\title{
BMJ
}

\section{Overall and cancer related mortality among patients with ocular inflammation treated with immunosuppressive drugs: retrospective cohort study}

\begin{abstract}
John H Kempen, associate professor of ophthalmology and epidemiology , 1,2,3 Ebenezer Daniel, instructor in ophthalmology, ${ }^{2,4}$ James P Dunn, associate professor of ophthalmology, ${ }^{4}$ C Stephen Foster, clinical professor of ophthalmology, ${ }^{6,7}$ Sapna Gangaputra, associate researcher in ophthalmology, ${ }^{4,8}$ Asaf Hanish, biostatistical programmer, ${ }^{3}$ Kathy J Helzlsouer, professor of epidemiology, ${ }^{5,9}$ Douglas A Jabs, professor of ophthalmology, ${ }^{4,5,10,11}$ R Oktay Kaçmaz, research fellow in ophthalmology, ${ }^{6,10}$ Grace A Levy-Clarke, ophthalmologist, ${ }^{12,13}$ Teresa L Liesegang, research coordinator, ${ }^{14}$ Craig W Newcomb, biostatistician, ${ }^{3}$ Robert B Nussenblatt, chief of laboratory of immunology, ${ }^{12}$ Siddharth S Pujari, research fellow in ophthalmology, ${ }^{6}$ James T Rosenbaum, professor of ophthalmology, medicine, and cell biology , ${ }^{14,15,16}$ Eric B Suhler, associate professor of ophthalmology, ${ }^{14,17}$ Jennifer E Thorne, associate professor of ophthalmology and epidemiology $\mathrm{y}^{4,5}$
\end{abstract}

\section{${ }^{1}$ Ocular Inflammation Service, Scheie Eye Institute, University of Pennsylvania, Philadelphia, PA, USA \\ ${ }^{2}$ Center for Preventive \\ Ophthalmology and Biostatistics, \\ Department of Ophthalmology, \\ University of Pennsylvania \\ ${ }^{3}$ Center for Clinical Epidemiology and Biostatistics, Department of Biostatistics and Epidemiology, \\ University of Pennsylvania \\ ${ }^{4}$ Department of Ophthalmology, \\ Johns Hopkins University, \\ Baltimore, MD, USA}

${ }^{5}$ Department of Epidemiology, Johns Hopkins University

${ }^{6}$ Massachusetts Eye Research and Surgery Institute, Cambridge, MA, USA

${ }^{7}$ Department of Ophthalmology, Harvard Medical School, Boston, MA, USA

${ }^{8}$ Fundus Photograph Reading Center, Department of

Ophthalmology, University of Wisconsin, Madison, WI, USA

${ }^{9}$ Center for Prevention and Research at Mercy, Mercy Medical Center, Baltimore

${ }^{10}$ Department of Ophthalmology, Mount Sinai School of Medicine, New York, NY, USA

${ }^{11}$ Department of Medicine, Mount Sinai School of Medicine

${ }^{12}$ Laboratory of Immunology, National Eye Institute, Bethesda, MD, USA

${ }^{13}$ St Luke's Cataract and Laser Institute, Tarpon Springs, FL, USA

${ }^{14}$ Department of Ophthalmology, Oregon Health and Science University, Portland, OR, USA

\section{ABSTRACT}

Context Whether immunosuppressive treatment adversely affects survival is unclear.

Objective To assess whether immunosuppressive drugs increase mortality.

Design Retrospective cohort study evaluating overall and cancer mortality in relation to immunosuppressive drug exposure among patients with ocular inflammatory diseases. Demographic, clinical, and treatment data derived from medical records, and mortality results from United States National Death Index linkage. The cohort's mortality risk was compared with US vital statistics using standardised mortality ratios. Overall and cancer mortality in relation to use or non-use of immunosuppressive drugs within the cohort was studied with survival analysis.

Setting Five tertiary ocular inflammation clinics. Patients 7957 US residents with non-infectious ocular inflammation, 2340 of whom received immunosuppressive drugs during follow up.

Exposures Use of antimetabolites, T cell inhibitors, alkylating agents, and tumour necrosis factor inhibitors. Main outcome measures Overall mortality, cancer mortality.

Results Over 66802 person years (17316 after exposure to immunosuppressive drugs), 936 patients died (1.4/ 100 person years), 230 (24.6\%) from cancer. For patients unexposed to immunosuppressive treatment, risks of death overall (standardised mortality ratio 1.02, 95\% confidence interval [CI] 0.94 to 1.11) and from cancer (1.10, 0.93 to 1.29) were similar to those of the US population. Patients who used azathioprine, methotrexate, mycophenolate mofetil, ciclosporin, systemic corticosteroids, or dapsone had overall and cancer mortality similar to that of patients who never took immunosuppressive drugs. In patients who used cyclophosphamide, overall mortality was not increased and cancer mortality was non-significantly increased. Tumour necrosis factor inhibitors were associated with increased overall (adjusted hazard ratio [HR] 1.99, 95\% Cl 1.00 to 3.98) and cancer mortality (adjusted HR 3.83, 1.13 to 13.01$)$.

Conclusions Most commonly used immunosuppressive drugs do not seem to increase overall or cancer mortality. Our results suggesting that tumour necrosis factor inhibitors might increase mortality are less robust than the other findings; additional evidence is needed.

\section{INTRODUCTION}

A common dilemma faced by patients with inflammatory diseases is whether the benefits of systemic immunosuppressive therapy warrant the associated risks. Of particular concern is the possibility of inducing a life threatening illness, such as cancer, in the process of achieving symptomatic benefits. Accurate information about the true extent of long term risks with such therapy is needed to inform clinical decision making for these patients. Observational studies - which provide most of the available evidence on the topic - are potentially subject to indications-for-treatment bias, because the conditions serving as the indication for immunosuppression are themselves often associated with an intrinsically higher risk of mortality or cancer, and the patients with more severe disease are more likely to receive the treatment. ${ }^{1}$

Available reports about the risk of mortality after immunosuppressive therapy outside the transplant setting are limited. A study of several hundred patients with rheumatoid arthritis treated with ciclosporin 
found no increased risk of mortality associated with this drug. ${ }^{2}$ One large observational study of patients with rheumatoid arthritis found no increased risk of overall mortality with tumour necrosis factor (TNF) inhibitors; ${ }^{3}$ another reported no increased risk of overall or cancer associated mortality. ${ }^{4}$ Findings of studies in patients with rheumatoid arthritis differ about whether use of corticosteroids increases ${ }^{5-8}$ or does not increase ${ }^{910}$ mortality risk.

More information is available about the risk of cancer after immunosuppressive treatment. ${ }^{1}$ The weight of evidence suggests that use of azathioprine and methotrexate for inflammatory diseases does not substantially increase the overall risk of cancer; the limited information about mycophenolate mofetil is also encouraging. Data about T cell inhibitors are limited outside the transplant setting. One report suggested no increased overall risk of cancer among patients with rheumatoid arthritis, ${ }^{2}$ whereas results in patients with psoriasis indicated a higher risk of leukaemia and of non-melanomatous skin cancers, ${ }^{11}$ which might be related to potentiation of the adverse effects of psoralen and ultraviolet A treatment by ciclosporin. ${ }^{1}$ Strong evidence exists for increased risk of skin, haematological, and (for cyclophosphamide) bladder cancers in connection with alkylating agents, ${ }^{1}$ and a few reports have indicated that mortality from all types of cancer is increased following treatment with such drugs. ${ }^{12}{ }^{13} \mathrm{~A}$ meta-analysis of patients with rheumatoid arthritis randomised to TNF inhibitor therapy in clinical trials found a 3.3-fold higher risk of cancer over 22-54 weeks' follow-up. ${ }^{14}$ However, several large observational studies following patients with rheumatoid arthritis over substantially longer periods ${ }^{35-17}$ and one study of patients with Crohn's disease found no increased overall risk of cancer. ${ }^{18}$

Non-infectious ocular inflammatory diseases provide a model system wherein the disease serving as the indication for immunosuppression seems unlikely to confer an increased risk of cancer or mortality, potentially avoiding the problem of indications-fortreatment bias. Here we report the primary results of a large scale retrospective cohort study evaluating the relation between immunosuppressive treatments and mortality in these patients - the Systemic Immunosuppressive Therapy for Eye diseases (SITE) cohort study.

\section{METHODS}

The SITE cohort study methods have been described in detail previously. ${ }^{19}$ Briefly, all eligible patients seen at five academic ocular inflammation practices in the United States during the years 1979-2005 contributed to this analysis. The time period was selected based on the years covered by the National Death Index at the time of the study. Eligible patients had a non-infectious ocular inflammatory diagnosis (uveitis, scleritis, cicatrising conjunctivitis of mucous membrane pemphigoid, and other conjunctival, corneal, optic nerve, and orbital inflammatory diseases). Patients known to have HIV infection were excluded. Patients diagnosed with cancer before cohort entry were excluded from the primary within cohort survival analyses, but were included for comparisons with the United States general population (which includes people with pre-existing cancer).

The centres directly managed immunosuppression in most instances, and kept detailed records that were available for review. Data about demographic, clinical, and treatment characteristics were obtained from medical records by a structured, protocol-driven review of every visit of every patient. At four centres, all eligible patients identified were studied. At the fifth centre, because of a larger volume of patients and limited resources, a random sample of about $40 \%$ of the eligible patients were studied, oversampling subgroups likely to have received immunosuppression and patients treated early in the period of observation, ${ }^{19}$ so as to maximise the information gained about immunosuppression and mortality. Reviews were done by five residency trained ophthalmologists with masters level or higher epidemiology training and one highly experienced ophthalmic technician and research coordinator. Use of immunosuppressive agents before cohort entry was noted along with the dosages of immunosuppressive agents and corticosteroids at all clinic visits. The broad range of additional data collection has been described previously. ${ }^{19}$ Quality control features built into the data system required immediate correction or verification of unlikely values. Records of patients who had been seen at more than one of the participating centres were merged.

Data on mortality incidence during 1979-2005 inclusive were obtained by linkage of patient identifiers to the US National Death Index, ${ }^{20}$ which provides near perfect ascertainment of mortality when US social security numbers are available (as in about $90 \%$ of our cohort). ${ }^{2122}$ Ascertainment of mortality using this approach is outstanding even based on the other identifiers we used. ${ }^{21}$ Perfect matches on social security number, all names, and date of birth were accepted as matches. Possible matches with inconsistencies or missing values in one or more of these fields were manually reviewed, and adjudicated by consensus.

Causes of death were obtained using the National Death Index "plus" feature, extracted from death certificates in the same manner as for US vital statistics, which has $96 \%$ code-recode reproducibility. ${ }^{23}$ Return of cancer codes (international classification of disease [ICD]-9 codes 140.0 to $208.9,239.0$ to 239.9 , or ICD10 codes $\mathrm{C} 00$ to $\mathrm{C} 97$ ) as the cause of death were taken as indicating a cancer death, within which return of lymphoma codes (ICD-9 codes 200.0 to 202.9 or ICD-10 codes $\mathrm{C} 81.0$ to $\mathrm{C} 85.9)$ were taken as indicating a lymphoma death.

The study was approved by the participating centres' institutional review boards, each of which approved waiver of consent for this retrospective study, and also was approved by the National Death Index review board. The study was conducted in compliance with the Declaration of Helsinki. 


\section{Statistical analysis}

Person time at risk of mortality was calculated beginning from 1 January 1979 or the first study centre visit (whichever was later) and ending at either the person's death or 31 December 2005, the last day on which death could have been detected. Person time before use of any immunosuppressive drug was counted with the unexposed group, whereas person time during or after use of an immunosuppressant was counted in that exposure group. Patients who were started on one or more additional immunosuppressive drugs continued to be counted as exposed to the first immunosuppressive drug to the end of follow-up, and also were counted as exposed to the additional drugs from the point of starting the additional drugs onwards. Cumulative and highest observed doses and duration of observed therapy were calculated.

To compare mortality in the cohort with that in the general population, US vital statistics mortality data (wonder.cdc.gov, accessed on 18 February 2009) were downloaded within strata of sex, race, and age (using five year age bands). Cohort data were sorted into the same strata, whence standardised mortality ratios (SMRs) and standardised cancer mortality ratios were calculated to make the comparison of mortality experience, adjusted for age, race, and sex.

For the within cohort survival analysis, Cox regression ${ }^{24}$ was used to adjust for potentially confounding variables: age, race, sex, smoking status, site of ocular inflammation, systemic inflammatory disease diagnoses, bilateral ocular inflammation, and other comorbidities as indicated by the Charlson index, which assigns points based on the presence and severity of systemic diseases. ${ }^{25}$ The categories presented in table 1 were used for categorical variables. Cox models were developed for each immunosuppressive agent, and for each class of agents, by forward stepwise selection among the covariates above (using the Wald $\chi^{2}$ statistic, $\mathrm{p} \leq 0.05$ ), but forcing the primary site of ocular inflammation into each model. Cox models evaluating quartiles and log transformed quantitative levels of both cumulative dose and highest observed dose also were constructed. All statistical analyses were done with SAS version 9.1 (SAS Corporation, Cary, NC).

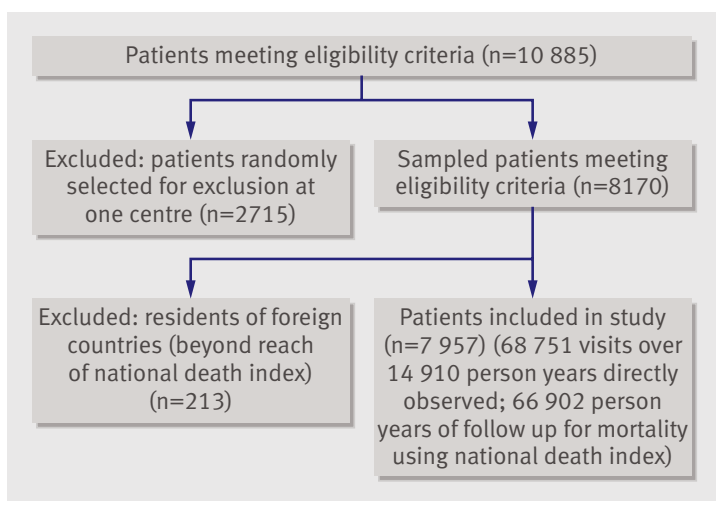

Fig 1 Study profile

\section{RESULTS}

During 1979-2005 inclusive, 10885 patients meeting eligibility criteria were seen at the five participating centres and identified by reviewing all charts (fig 1). At the largest centre, where a random sample of patients was studied, 2715 were not sampled, and hence were excluded. Two hundred and thirteen more were resident outside the United States, beyond the reach of the National Death Index. The remaining 7957 were observed over 68751 visits, spanning 14910 person years at the clinical centres. Of these, 2340 were treated with immunosuppressive drugs during observation at the clinical centres. Follow-up for mortality using the National Death Index to the end of 2005 covered 66802 person years, including 49486 person years unexposed to immunosuppressive drugs and 17316 person years of time after exposure to immunosuppressants.

Table 1 shows presenting characteristics of the cohort. Because the analysis of exposure was time dependent, with patients moving from the unexposed to exposed groups, fixed characteristics of each patient who contributed person time to each exposure category are included in every category to which they contributed. The median age at presentation was older among patients who were treated with alkylating agents (53.8 years) and dapsone (70.5 years), drugs that were often used for mucous membrane pemphigoid with ocular involvement (usually a disease of the elderly). The distribution of sexes across treatment groups reflected the greater frequency of ocular inflammation among females than among males. The frequency of treatment with antimetabolites and $\mathrm{T}$ cell inhibitors was similar across racial and ethnic groups, but white people were more commonly treated with alkylating agents, dapsone, and TNF inhibitors than other groups. Smoking status was similar across most treatment groups, but smoking was less common among patients treated with dapsone. Co-morbidity, as indicated by the Charlson index, was greatest in patients treated with TNF inhibitors or alkylating agents, and was least in patients who did not receive immunosuppression. Omitting mucous membrane pemphigoid itself, systemic inflammatory diseases were associated with ocular inflammation in 1986 patients $(25.0 \%)$, the most common conditions being sarcoidosis (5.6\%), juvenile idiopathic arthritis (3.8\%), ankylosing spondylitis (3.7\%), and rheumatoid arthritis $(3.4 \%)$ (see supplemental table 1). Systemic inflammatory disease co-morbidity was less frequent among patients who never received immunosuppressive drugs $(21.9 \%)$ than in those who were exposed to the drugs. Nevertheless, only a minority of patients who were exposed to the drugs had associated systemic inflammatory diseases - with the exception of those who received TNF inhibitors (74.2\%).

During follow-up, 936 deaths occurred, 230 (24.6\%) of which were attributed to cancer. Of these, 323 deaths occurred in patients exposed to immunosuppressive drugs and 613 in unexposed patients. The cohort's overall mortality risk - adjusted only for age, 


\section{Table 1|Demographic and clinical characteristics}

\begin{tabular}{|c|c|c|c|c|c|c|c|}
\hline & Antimetabolites & T cell inhibitors & $\begin{array}{l}\text { Alkylating } \\
\text { agents }\end{array}$ & TNF inhibitors & Dapsone & None & All patients* \\
\hline Number of patients contributing person time & 1634 & 775 & 486 & 213 & 255 & 6819 & 7957 \\
\hline Age, years; median (range) & $45.4(2.8-93.6)$ & $37.8(3.2-86.3)$ & $53.8(5.4-91.4)$ & $41.4(3.2-79.2)$ & $70.5(14.0-92.4)$ & $43.6(2.1-96.9)$ & $43.9(2.1-96.9)$ \\
\hline \multicolumn{8}{|l|}{ Sex } \\
\hline Male & $559(34.2)$ & $305(39.4)$ & $208(42.8)$ & $77(36.2)$ & $93(36.5)$ & $2534(37.2)$ & $2961(37.2)$ \\
\hline Female & $1075(65.8)$ & $470(60.6)$ & $278(57.2)$ & $136(63.8)$ & $162(63.5)$ & $4285(62.8)$ & $4996(62.8)$ \\
\hline \multicolumn{8}{|l|}{ Race } \\
\hline White & $1276(78.1)$ & $587(75.7)$ & $404(83.1)$ & $173(81.2)$ & $238(93.3)$ & $5068(74.3)$ & $5972(75.1)$ \\
\hline Black/African American & $185(11.3)$ & $110(14.2)$ & $46(9.5)$ & $14(6.6)$ & $5(2.0)$ & $969(14.2)$ & $1077(13.5)$ \\
\hline Hispanic & $66(4.0)$ & $26(3.4)$ & $15(3.1)$ & $12(5.6)$ & $9(3.5)$ & $204(3.0)$ & $246(3.1)$ \\
\hline Other & $68(4.2)$ & $37(4.8)$ & $15(3.1)$ & $13(6.1)$ & $3(1.2)$ & $284(4.2)$ & 339 (4.3) \\
\hline Missing & $39(2.4)$ & $15(1.9)$ & $6(1.2)$ & $1(0.47)$ & 0 & $294(4.3)$ & $323(4.1)$ \\
\hline \multicolumn{8}{|l|}{ Smoking } \\
\hline Never & $965(59.1)$ & $370(47.7)$ & $266(54.7)$ & $125(58.7)$ & $162(63.5)$ & $3915(57.4)$ & $4551(57.2)$ \\
\hline Former & $207(12.7)$ & $85(11.0)$ & $69(14.2)$ & $31(14.6)$ & $21(8.2)$ & $874(12.8)$ & $1020(12.8)$ \\
\hline Current & $254(15.5)$ & $165(21.3)$ & $83(17.1)$ & $33(15.5)$ & $12(4.7)$ & 1194 (17.5) & $1363(17.1)$ \\
\hline Missing & $208(12.7)$ & $155(20.0)$ & $68(14.0)$ & $24(11.3)$ & $60(23.5)$ & $836(12.3)$ & $1023(12.9)$ \\
\hline \multicolumn{8}{|l|}{ Charlson index ${ }^{25}$ score } \\
\hline 0 & $724(44.4)$ & $392(50.6)$ & $173(35.6)$ & $56(26.3)$ & 87 (34.1) & $4291(63.0)$ & $4781(60.1)$ \\
\hline 1 & $502(30.8)$ & $201(26.0)$ & $142(29.2)$ & $95(44.6)$ & $74(29.0)$ & $1371(20.1)$ & $1730(21.8)$ \\
\hline$\geq 2$ & $406(24.9)$ & $181(23.4)$ & $171(35.2)$ & $62(29.1)$ & $94(36.9)$ & $1153(16.9)$ & $1441(18.1)$ \\
\hline Diagnosis with malignancy before cohort entry $\dagger$ & $71(4.3)$ & $19(2.5)$ & $29(6.0)$ & $7(3.3)$ & $17(6.7)$ & $327(4.8)$ & $374(4.7)$ \\
\hline \multicolumn{8}{|l|}{ Type of ocular inflammation } \\
\hline Anterior uveitis & $368(22.5)$ & $137(17.7)$ & $33(6.8)$ & $68(31.9)$ & $3(1.2)$ & $2626(38.5)$ & $2877(36.2)$ \\
\hline Intermediate uveitis & $270(16.5)$ & $194(25.0)$ & $24(4.9)$ & $32(15.0)$ & $6(2.4)$ & $1490(21.9)$ & $1655(20.8)$ \\
\hline Posterior or panuveitis & $517(31.6)$ & $341(44.0)$ & $114(23.5)$ & $45(21.1)$ & $7(2.7)$ & $1500(22.0)$ & $1831(23.0)$ \\
\hline Scleritis & $245(15.0)$ & $55(7.1)$ & $99(20.4)$ & $56(26.3)$ & $7(2.7)$ & $769(11.3)$ & $937(11.8)$ \\
\hline Ocular mucous membrane pemphigoid & $161(9.9)$ & $25(3.2)$ & $165(34.0)$ & $3(1.4)$ & $227(89.0)$ & $282(4.1)$ & $453(5.7)$ \\
\hline Othert & $73(4.5)$ & $23(3.0)$ & $51(10.5)$ & $9(4.2)$ & $5(2.0)$ & $152(2.2)$ & $204(2.6)$ \\
\hline Bilateral ocular inflammation & $1360(83.2)$ & $681(87.9)$ & $396(81.5)$ & $171(80.3)$ & $243(95.3)$ & $4731(69.4)$ & $5677(71.4)$ \\
\hline Systemic inflammatory diseases§ & $648(39.7)$ & $235(30.3)$ & $185(38.1)$ & $158(74.2)$ & $33(12.9)$ & $1491(21.9)$ & $1986(25.0)$ \\
\hline Follow-up time for mortality, years: median (IQR) & $4.8(2.44-8.2)$ & $7.6(3.57-13.7)$ & $7.4(3.63-12.5)$ & $2.8(1.68-4.2)$ & $8.8(4.51-13.5)$ & $5.9(2.08-11.4)$ & $7.1(3.59-12.2)$ \\
\hline Follow-up time for mortality, person years & 9695 & 7059 & 4222 & 692 & 2450 & 49486 & 66802 \\
\hline Follow-up time at clinics, person years & 3885 & 2500 & 1779 & 360 & 891 & 8431 & 14810 \\
\hline Total deaths & 169 & 73 & 125 & 11 & 101 & 613 & 936 \\
\hline $\begin{array}{l}\text { Duration of immunosuppressive treatment, years; } \\
\text { median (IQR) }\end{array}$ & $1.4(0.5-2.7)$ & $1.5(0.4-3.5)$ & $0.9(0.3-1.6)$ & $1.2(0.4-2.0)$ & $0.7(0.2-2.5)$ & & \\
\hline \multicolumn{8}{|l|}{ Use of other classes of immunosuppressive drugs } \\
\hline 0 classes used & 0 & 0 & 0 & 0 & 0 & $5617(82.4)$ & $5617(70.7)$ \\
\hline 1 class used & $906(55.4)$ & $322(41.5)$ & $176(36.2)$ & $30(14.1)$ & $90(35.3)$ & $863(12.7)$ & $1524(19.2)$ \\
\hline 2 classes used & $545(33.4)$ & $323(41.7)$ & $167(34.4)$ & $101(47.4)$ & $102(40.0)$ & 264 (3.9) & $619(7.8)$ \\
\hline 3 classes used & $153(9.4)$ & $102(13.2)$ & $113(23.3)$ & $63(29.6)$ & 49 (19.2) & $68(1.00)$ & $160(2.0)$ \\
\hline 4 classes used & $29(1.8)$ & $27(3.5)$ & $29(6.0)$ & $18(8.5)$ & $13(5.1)$ & $7(0.10)$ & $29(0.36)$ \\
\hline 5 classes used & $1(0.06)$ & $1(0.13)$ & $1(0.21)$ & $1(0.47)$ & $1(0.39)$ & 0 & $1(0.01)$ \\
\hline
\end{tabular}

$\mathrm{IQR}=$ interquartile range. Data are number $(\%)$ unless otherwise stated.

*Because the analysis of exposure to immunosuppressive agents was time dependent-with patients transitioning from unexposed to exposed (see statistical methods)—fixed

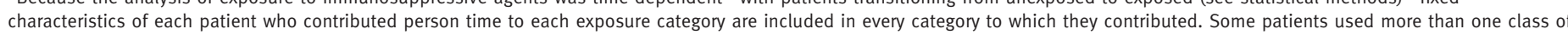
immunosuppressive drug during follow-up.

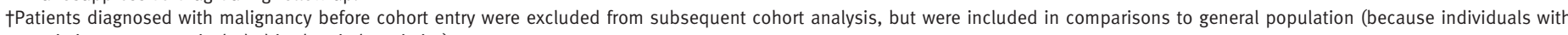
pre-existing cancer are included in the vital statistics).

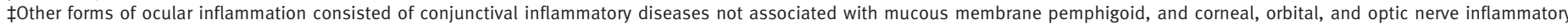
diseases.

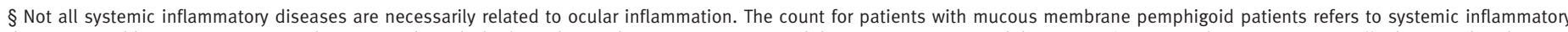

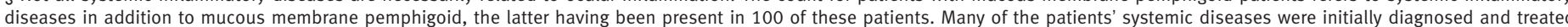

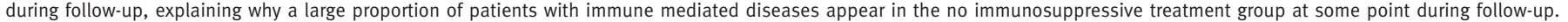

ПBecause use of immunosuppressive therapy was coded as a time dependent variable, some patients contributing person time to the "unexposed" group went on to receive

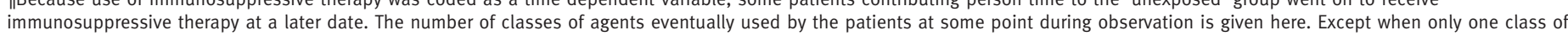
drug was used, the sum of patients in the immunosuppressive drug columns will not add up to the number in the all patients column. Seven patients treated with intravenous

immunoglobulin and none of the other immunosuppressive drugs are not included in any of these categories. 
Table 2 | Risk of overall and cancer mortality in cohort compared with 1999-2005 US general population, standardised for age, race, and sex

\begin{tabular}{|c|c|c|c|c|c|c|c|c|c|}
\hline $\begin{array}{l}\text { Use of immuno- } \\
\text { suppressive agents }\end{array}$ & $\begin{array}{l}\text { Number } \\
\text { of } \\
\text { patients }\end{array}$ & $\begin{array}{l}\text { Median } \\
\text { follow-up } \\
\text { time }\end{array}$ & $\begin{array}{l}\text { Observed } \\
\text { deaths }\end{array}$ & $\begin{array}{l}\text { Total } \\
\text { person } \\
\text { time }\end{array}$ & $\begin{array}{c}\text { Indirectly } \\
\text { standardised } \\
\text { mortality rate per } \\
100 \text { person years }\end{array}$ & $\begin{array}{l}\text { Standardised } \\
\text { mortality ratio } \\
(95 \% \mathrm{Cl})\end{array}$ & $\begin{array}{l}\text { Cancer } \\
\text { deaths }\end{array}$ & $\begin{array}{c}\text { Indirectly } \\
\text { standardised cancer } \\
\text { mortality rate per } \\
100 \text { person years }\end{array}$ & $\begin{array}{c}\text { Standardised cancer } \\
\text { mortality ratio } \\
(95 \% \mathrm{Cl})\end{array}$ \\
\hline None & 6545 & 5.68 & 562 & 46086 & 0.89 & $1.02(0.94$ to 1.11$)$ & 149 & 0.22 & 1.10 (0.93 to 1.29$)$ \\
\hline Any & 2285 & 5.60 & 321 & 17006 & 0.91 & $1.04(0.93$ to 1.16$)$ & 69 & 0.20 & 1.03 (0.80 to 1.31$)$ \\
\hline Total & 7628 & 6.98 & 883 & 63079 & 0.90 & $1.03(0.96$ to 1.10$)$ & 218 & 0.22 & 1.07 (0.94 to 1.23$)$ \\
\hline
\end{tabular}

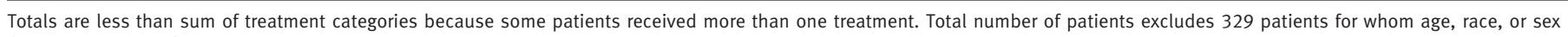

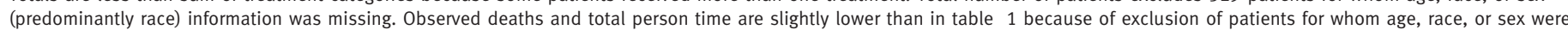
missing for standardised mortality ratio calculations.

sex, and race - was similar to that for the US population, both for patients unexposed to immunosuppressive therapy (standardised mortality ratio [SMR] 1.02, $95 \%$ confidence interval [CI] 0.94 to 1.11 ; table 2 ) and for the cohort as a whole (1.03, 0.96 to 1.10$)$. Similarly, overall cancer mortality was similar to that of the US population both for cohort members unexposed to immunosuppressive drugs (cancer specific SMR 1.10, $95 \%$ CI 0.93 to 1.29 ) and for the whole cohort (1.07, 0.94 to 1.23$)$.

The relation of immunosuppressive therapy to overall and cancer mortality is summarised in tables 3 and 4 (see also fig 2 and fig 3). Non-treatment factors that were consistently associated with increased mortality risk in the within cohort comparisons (and were adjusted for in the models) included increasing age, African-American race, higher Charlson index score, active smoking, and a diagnosis of Wegener's granulomatosis or rheumatoid arthritis. A supplemental table compares overall and cancer mortality among treated cohort members with that of the US population using SMRs (see supplemental table 2), but these findings are less reliable because vital statistics data only included information on age, sex, and race, and not the remaining confounding factors.

After adjusting for confounding, the antimetabolites as a class of immunosuppressive drugs were not associated with a substantial increase in overall mortality (fully adjusted hazard ratio $1.08,95 \%$ CI 0.86 to 1.37 ) or cancer mortality $(0.89,0.54$ to 1.48$)$. The most commonly used antimetabolites-azathioprine, methotrexate, and mycophenolate mofetil-were not individually associated with increased risk of overall or cancer mortality either. The tendency towards increased crude and demographic adjusted hazard ratios observed with antimetabolite therapy (see tables 3 and 4) corresponded to greater use of these drugs in patients who had systemic inflammatory comorbidities and were older.

Likewise, the fully adjusted within cohort comparison found no increased overall mortality risk associated with $\mathrm{T}$ cell inhibitors as a class (fully adjusted hazard ratio $0.81,95 \%$ CI 0.59 to 1.11 ). Cancer mortality was not significantly increased with $\mathrm{T}$ cell inhibitor therapy either $(0.78,0.38$ to 1.59$)$. Overall and cancer mortality results for ciclosporin individually had a pattern similar to that for $\mathrm{T}$ cell inhibitors in general.
Alkylating agents as a class were not associated with significantly increased overall mortality in the within cohort comparisons (fully adjusted hazard ratio 1.17, 95\% CI 0.85 to 1.61 ), but all-cancer mortality was nonsignificantly raised $(1.74,0.91$ to 3.32$)$. Comparison of overall and cancer mortality with that of the general US population gave similar results (SMR 1.06, 95\% CI 0.88 to 1.26 ; cancer-specific SMR 1.42, 95\% CI 0.99 to 1.98). Findings for cyclophosphamide followed similar patterns to those for alkylating agents as a class. Only 87 patients had been treated with chlorambucil, limiting statistical precision for evaluation of its association with overall and cancer mortality.

TNF inhibitors in aggregate were associated with significant increases both in overall mortality (fully adjusted hazard ratio $1.99,95 \%$ CI 1.00 to 3.98 ) and cancer mortality (3.83, 95\% CI: 1.13 to 13.01$)$. Comparisons with mortality in the US general populationadjusting only for age, sex, and race-yielded similar results for both overall (SMR 2.62, 95\% CI 1.30 to 4.71) and cancer mortality (cancer-specific SMR 4.05, 95\% CI 1.28 to 9.53). Estimated risk ratios for etanercept and infliximab were similar in magnitude, but were non-significant, with a smaller number of observations. Little information was available about adalimumab, which was introduced shortly before 2005 . Sensitivity analyses eliminating patients with systemic inflammatory co-morbidities did not substantially alter the SMR for TNF inhibitors, but because all but two deaths (neither of which was from cancer) were eliminated in this sensitivity analysis, confidence intervals became very wide. The 11 deaths observed in the patients on TNF inhibitors were all attributed to different causes. Six of the patients who died after taking TNF inhibitors had also taken alkylating agents during observation; formal interaction testing gave some suggestion of an interaction, but the result was not significant (fully adjusted hazard ratio for interaction 2.24, 95\% CI 0.90 to 5.64 ).

Systemic corticosteroid therapy was not associated with increased overall (hazard ratio 1.13, 95\% CI 0.96 to 1.33$)$ or cancer mortality $(1.02,0.72$ to 1.45$)$ after adjusting for confounding. Patients exposed to dapsone likewise had no significant change in overall $(0.92,0.55$ to 1.55$)$ or cancer mortality $(0.92,0.28$ to $2.99)$ in the fully adjusted within cohort analyses. Evaluation of potential associations between immunosuppressive drugs and lymphoma mortality risk showed no significant association, but these analyses had low 
power because there were only nine lymphoma deaths among immunosuppressed individuals in this cohort.

Evaluation of dose-response relations for individual antimetabolites, $\mathrm{T}$ cell inhibitors, alkylating agents, dapsone, and systemic corticosteroids did not show increasing risks of overall or cancer mortality with increasing cumulative dose or across a dose threshold, or with increasing highest observed dose. The data were insufficient to evaluate these relations for TNF inhibitors.

Within cohort survival analyses excluding patients with systemic inflammatory diseases gave similar results (see supplemental tables 3 and 4), except that data were not sufficient to conduct these analyses meaningfully for TNF inhibitors. Additional sensitivity analyses undertaken were: (1) exclusion of subsets of calendar time to address potential cohort effects; (2) exclusion of the first four years of follow-up to allow for a potential lag in the occurrence of adverse effects of

\begin{tabular}{|c|c|c|}
\hline & $\begin{array}{c}\text { Hazard ratio } \\
(95 \% \mathrm{Cl})\end{array}$ & $\begin{array}{c}\text { Hazard ratio } \\
(95 \% \mathrm{Cl})\end{array}$ \\
\hline Azathioprine & & 0.99 (0.72 to 1.38$)$ \\
\hline Methotrexate & - & $1.02(0.78$ to 1.34$)$ \\
\hline Mycophenolate mofetil & - & 0.90 (0.48 to 1.68$)$ \\
\hline Antimetabolite (in aggregate) & - & 1.08 (0.86 to 1.37$)$ \\
\hline Ciclosporin & & $0.79(0.57$ to 1.10$)$ \\
\hline T cell inhibitor (in aggregate) & & $0.81(0.59$ to 1.11$)$ \\
\hline Chlorambucil & & $1.43(0.72$ to 2.85$)$ \\
\hline Cyclophosphamide & & 1.14 (0.81 to 1.60$)$ \\
\hline Alkylating agent (in aggregate) & ) & 1.17 (0.85 to 1.61$)$ \\
\hline Etanercept & & 2.18 (0.93 to 5.09$)$ \\
\hline Infliximab & & 2.44 (0.90 to 6.62$)$ \\
\hline TNF inhibitor (in aggregate) & & 1.99 (1.00 to 3.98$)$ \\
\hline Dapsone & - & $0.92(0.55$ to 1.55$)$ \\
\hline Systemic corticosteroids & - & 1.13 (0.96 to 1.33$)$ \\
\hline
\end{tabular}

Fig 2 | Adjusted relative hazard of all cause mortality for each immunosuppressive agent and class of agents studied

$\begin{array}{lll} & \begin{array}{l}\text { Hazard ratio } \\ (\mathbf{9 5 \%} \mathbf{~ C l})\end{array} & \begin{array}{c}\text { Hazard ratio } \\ \mathbf{( 9 5 \%} \mathbf{~ C l})\end{array} \\ \text { Azathioprine } & 1.13(0.60 \text { to } 2.14) \\ \text { Methotrexate } & 0.89(0.48 \text { to } 1.63) \\ \text { Mycophenolate mofetil } & 0.83(0.20 \text { to } 3.52) \\ \text { Antimetabolite (in aggregate) } \\ \text { Ciclosporin }\end{array}$

Fig $3 \mid$ Adjusted relative hazard of mortality attributed to cancer for each immunosuppressive agent and class of agents studied treatment; (3) exclusion of patients who began followup after 1997 (to address potential concerns that slow growing tumours may not have caused death during the period of observation); and (4) exclusion of patients with mucous membrane pemphigoid and intermediate uveitis, who had SMRs significantly different from 1.0 (see supplemental table 2). None of these sensitivity analyses resulted in qualitatively different conclusions, and risk ratios were quite similar to those reported above, except that overall and cancer mortality risk ratios tended to be closer to 1 for patients treated with cyclophosphamide before 1998. The available data were insufficient to perform sensitivity analyses (2) to (4) for TNF inhibitors or to perform sensitivity analysis (4) for mycophenolate mofetil.

\section{DISCUSSION}

This retrospective cohort study of 7957 patients with ocular inflammation, 2340 of whom were treated with immunosuppressive drugs, found no significantly increased risk of death or of death from cancer among those who had received treatment with antimetabolites (predominantly methotrexate, azathioprine, and mycophenolate mofetil), $\mathrm{T}$ cell inhibitors (predominantly ciclosporin), dapsone, or systemic corticosteroids, after adjusting for confounding factors. Treatment with cyclophosphamide was not associated with significant increases in overall mortality, but there was a non-significant suggestion of increased cancer mortality. Data about treatment with TNF inhibitors were less methodologically robust than those for other drugs, but observations of significant increases in overall mortality and cancer mortality after TNF inhibitor therapy add to concerns about these treatments, especially in patients also treated with alkylating agents, and require further investigation.

\section{Strengths and weaknesses of the study design}

A principal strength of this study was that the eye disease indication for use or non-use of immunosuppression was a non-lethal disease, overcoming the causal pathway of indications-for-treatment bias as effectively as possible outside a randomised trial, which is not a feasible study design option to address this question. ${ }^{19}$ Indications-for-treatment bias has been a relevant concern for most previous studies on this topic. ${ }^{1}$ Direct comparison with the US vital statistics verified that patients in this group who were free of systemic inflammatory diseases did not have a significantly increased risk of overall mortality or cancer mortality. Thus, the study methodology does seem to avoid the problem of indications-for-treatment bias. Concerns about entering both prevalent and incident cases into the study cohort also are minimised when the disease condition does not affect the risk of the study outcome.

Ascertainment of overall and cancer mortality using the National Death Index was probably excellent. ${ }^{20-22}$ The duration of follow-up after exposure was favourable for all agents except mycophenolate mofetil and the TNF inhibitors (the latter of which were associated with increased risk despite limited follow-up time); 
Table 3 |Use of immunosuppressive drugs and risk of overall mortality*

\begin{tabular}{|c|c|c|c|c|c|c|}
\hline Agent & Crude HR $(95 \% \mathrm{Cl})$ & $\mathbf{P}$ & $\begin{array}{l}\text { HR adjusted for age, } \\
\text { race, sex }(95 \% \mathrm{Cl})\end{array}$ & $P$ & $\begin{array}{l}\text { HR fully adjusted } \\
\text { model }(95 \% \mathrm{Cl})\end{array}$ & $P$ \\
\hline No immunosuppressive agent & 1.00 & & 1.00 & & 1.00 & \\
\hline Antimetabolite (any) & $1.60(1.33$ to 1.91$)$ & $<0.0001$ & 1.23 (1.02 to 1.47$)$ & 0.029 & 1.08 (0.86 to 1.37$)$ & 0.50 \\
\hline Azathioprine & $1.73(1.35$ to 2.21$)$ & $<0.0001$ & $1.13(0.88$ to 1.46$)$ & 0.33 & $0.99(0.72$ to 1.38$)$ & 0.97 \\
\hline Methotrexate & $1.56(1.25$ to 1.95$)$ & $<0.0001$ & $1.19(0.95$ to 1.49$)$ & 0.129 & $1.02(0.78$ to 1.34$)$ & 0.87 \\
\hline Mycophenolate mofetil & 0.94 (0.53 to 1.67$)$ & 0.82 & $1.00(0.56$ to 1.78$)$ & 0.99 & 0.90 (0.48 to 1.68$)$ & 0.73 \\
\hline T cell inhibitor (any) & $0.89(0.69$ to 1.14$)$ & 0.35 & $1.22(0.95$ to 1.56$)$ & 0.121 & $0.81(0.59$ to 1.11$)$ & 0.18 \\
\hline Ciclosporin & 0.81 (0.63 to 1.05$)$ & 0.118 & 1.17 (0.90 to 1.52$)$ & 0.25 & $0.79(0.57$ to 1.10$)$ & 0.16 \\
\hline Alkylating agent (any) & 2.36 (1.92 to 2.90$)$ & $<0.0001$ & $1.26(1.02$ to 1.56$)$ & 0.031 & $1.17(0.85$ to 1.61$)$ & 0.34 \\
\hline Chlorambucil & $1.33(0.73$ to 2.41$)$ & 0.35 & 1.97 (1.08 to 3.59) & 0.027 & $1.43(0.72$ to 2.85$)$ & 0.30 \\
\hline Cyclophosphamide & $2.54(2.05$ to 3.14$)$ & $<0.0001$ & 1.19 (0.96 to 1.49$)$ & 0.116 & $1.14(0.81$ to 1.60$)$ & 0.45 \\
\hline TNF inhibitor (any) & $1.45(0.75$ to 2.82$)$ & 0.27 & 1.96 (1.01 to 3.81$)$ & 0.048 & 1.99 (1.00 to 3.98) & 0.050 \\
\hline Etanercept & 1.78 (0.79 to 3.99$)$ & 0.16 & 2.04 (0.91 to 4.59) & 0.085 & 2.18 (0.93 to 5.09$)$ & 0.072 \\
\hline Infliximab & $1.31(0.49$ to 3.51$)$ & 0.59 & 2.25 (0.83 to 6.05$)$ & 0.110 & 2.44 (0.90 to 6.62$)$ & 0.080 \\
\hline Dapsone & $3.45(2.76$ to 4.30$)$ & $<0.0001$ & $0.98(0.77$ to 1.24$)$ & 0.85 & $0.92(0.55$ to 1.55$)$ & 0.77 \\
\hline No systemic corticosteroids & 1.00 & & 1.00 & & 1.00 & \\
\hline Systemic corticosteroids & $1.03(0.90$ to 1.19$)$ & 0.63 & 1.24 (1.08 to 1.43$)$ & 0.003 & $1.13(0.96$ to 1.33$)$ & 0.15 \\
\hline \multicolumn{7}{|c|}{$\begin{array}{l}\text { HR=hazard ratio; } \mathrm{Cl}=\text { confidence interval; TNF=tumour necrosis factor. } \\
\text { *For immunosuppressive agents, each comparison is of person time after exposure to the agent indicated compared with patients never exposed to } \\
\text { any of the agents listed. For corticosteroids, the comparison is of person time after use of systemic corticosteroids versus person time before use of } \\
\text { systemic corticosteroids. Fully adjusted models adjust for age, race, sex, smoking status, site of ocular inflammation, bilaterality of ocular } \\
\text { inflammation, Charlson index score, and indicator variables for those systemic inflammatory diseases that were significantly associated with mortality } \\
\text { in Cox regression. In addition to the agents listed, small numbers of patients taking leflunomide, tacrolimus, sirolimus, and adalimumab were } \\
\text { included in the antimetabolite, T cell inhibitor, and TNF inhibitor groups, respectively. }\end{array}$} \\
\hline
\end{tabular}

exclusion of patients with less than eight years' follow up for mortality did not increase risk ratios, suggesting that the amount of follow-up time was sufficient to ascertain overall and cancer mortality for the other drugs. Because deaths from cancer are typically recognised easily, and because the proportion of mortality attributed to cancer was as expected, any effect of miscoding of death certificates on cancer mortality results was probably small.

Although the study's ascertainment of immunosuppressive drug use (typically administered by the clinics) was probably excellent during follow-up at the clinics, some drug exposures before or after the period of management at participating clinics may

\begin{tabular}{|c|c|c|c|c|c|c|}
\hline Agent & Crude HR $(95 \% \mathrm{Cl})$ & $\mathrm{P}$ & $\begin{array}{l}\text { HR adjusted for age, } \\
\text { race, sex }(95 \% \mathrm{Cl})\end{array}$ & $P$ & $\begin{array}{l}\text { HR fully adjusted } \\
\text { model }(95 \% \mathrm{Cl})\end{array}$ & $\mathbf{P}$ \\
\hline No immunosuppressive drug & 1.00 & & 1.00 & & 1.00 & \\
\hline Antimetabolite (any) & $1.16(0.76$ to 1.76$)$ & 0.49 & 0.87 (0.57 to 1.32$)$ & 0.50 & 0.89 (0.54 to 1.48$)$ & 0.66 \\
\hline Azathioprine & $1.73(1.04$ to 2.87$)$ & 0.034 & $1.06(0.63$ to 1.77$)$ & 0.83 & $1.13(0.60$ to 2.14$)$ & 0.70 \\
\hline Methotrexate & $1.03(0.60$ to 1.76$)$ & 0.93 & 0.76 (0.44 to 1.32$)$ & 0.33 & $0.89(0.48$ to 1.63$)$ & 0.70 \\
\hline Mycophenolate mofetil & 0.65 (0.16 to 2.66$)$ & 0.55 & $0.67(0.16$ to 2.76$)$ & 0.58 & 0.83 (0.20 to 3.52$)$ & 0.80 \\
\hline T cell inhibitor (any) & $0.85(0.51$ to 1.44$)$ & 0.55 & 1.15 (0.68 to 1.95$)$ & 0.60 & $0.78(0.38$ to 1.59$)$ & 0.50 \\
\hline Ciclosporin & $0.88(0.52$ to 1.48$)$ & 0.63 & 1.24 (0.73 to 2.10$)$ & 0.42 & 0.82 (0.40 to 1.67$)$ & 0.59 \\
\hline Alkylating agent (any) & $2.36(1.54$ to 3.60$)$ & $<0.0001$ & 1.21 (0.78 to 1.88$)$ & 0.39 & 1.74 (0.91 to 3.32$)$ & 0.092 \\
\hline Chlorambucil & $1.02(0.25$ to 4.14$)$ & 0.97 & 1.54 (0.38 to 6.27$)$ & 0.55 & 2.29 (0.53 to 9.83$)$ & 0.26 \\
\hline Cyclophosphamide & 2.54 (1.64 to 3.93$)$ & $<0.0001$ & 1.14 (0.72 to 1.79$)$ & 0.58 & 1.61 (0.81 to 3.22$)$ & 0.17 \\
\hline TNF inhibitor (any) & $2.06(0.65$ to 6.55$)$ & 0.22 & $2.44(0.77$ to 7.75$)$ & 0.132 & $3.83(1.13$ to 13.01$)$ & 0.031 \\
\hline Etanercept & $2.47(0.60$ to 10.06$)$ & 0.21 & $2.51(0.61$ to 10.24$)$ & 0.20 & 4.38 (0.96 to 19.93$)$ & 0.056 \\
\hline Infliximab & $1.42(0.20$ to 10.26$)$ & 0.73 & $2.13(0.29$ to 15.51$)$ & 0.45 & 2.95 (0.40 to 21.83$)$ & 0.29 \\
\hline Dapsone & 1.92 (1.06 to 3.47$)$ & 0.031 & 0.55 (0.29 to 1.02$)$ & 0.056 & $0.92(0.28$ to 2.99$)$ & 0.89 \\
\hline No systemic corticosteroids & 1.00 & & 1.00 & & 1.00 & \\
\hline Systemic corticosteroids & 0.95 (0.70 to 1.28$)$ & 0.72 & 1.10 (0.81 to 1.49$)$ & 0.55 & $1.02(0.72$ to 1.45$)$ & 0.89 \\
\hline \multicolumn{7}{|c|}{$\begin{array}{l}\text { HR=hazard ratio; } \mathrm{Cl}=\text { confidence interval; TNF=tumour necrosis factor. } \\
\text { *For immunosuppressive drugs, each comparison is of person time after exposure to the agent compared with patients never exposed to any of the } \\
\text { agents listed. For corticosteroids, the comparison is of person time after use of systemic corticosteroids versus person time before use of systemic } \\
\text { corticosteroids. Fully adjusted models adjust for age, race, sex, smoking status, site of ocular inflammation, bilaterality of ocular inflammation, } \\
\text { Charlson index score, and indicator variables for those systemic inflammatory diseases that were significantly associated with mortality in Cox } \\
\text { regression. In addition to the agents listed, small numbers of patients taking leflunomide, tacrolimus, sirolimus, and adalimumab were included in } \\
\text { the antimetabolite, T cell inhibitor, and TNF inhibitor groups, respectively. }\end{array}$} \\
\hline
\end{tabular}


have been missed. Had such misclassification been sufficient to mask a true association between drug exposure and mortality, a substantially increased overall mortality among the unexposed cohort members compared with the United States general population would be expected, but no such pattern was observed (table 2).

For most drugs, a large sample size and a substantial length of follow-up for exposed and unexposed patients allowed the conduct of a broad array of doseresponse relation analyses and sensitivity analyses, each of which supported the primary conclusions. However, for mycophenolate mofetil, chlorambucil, and TNF inhibitors the available information was less extensive. Additional follow up or more observations would be valuable for evaluation of these drugs. For the TNF inhibitor analyses in particular, dose-response and analyses excluding the first four years of follow-up or requiring long term follow-up were inadequate because of the limited extent of observations regarding these newer drugs. However, such analyses are generally used to unmask effects of high doses or remove possible "immortal person time" from an analysis, which would usually increase rather than diminish risk ratios.

\section{Implications of the study}

Patients with ocular inflammation who take azathioprine, methotrexate, ciclosporin, dapsone, and systemic corticosteroids most likely do not increase their risk of mortality or cancer mortality substantially. Longer term follow-up of the cohort would be valuable to obtain more data about mycophenolate mofetil, for which the available follow-up time is currently limited, but the available data do not suggest increased overall or cancer mortality with this agent. These conclusions should be generalisable to other disease indications for immunosuppressive treatment unless a disease-treatment interaction that increases the risk of death exists for other diseases, which seems unlikely.

We are not aware of previous reports regarding mortality risk for most of the agents studied. However, our negative results are broadly consistent with published work on risk of cancer for the antimetabolites azathioprine, methotrexate, and mycophenolate mofetil. ${ }^{1}$ One of two previous reports suggested an increased risk of leukaemia and skin cancers (among many cancers evaluated) with ciclosporin in patients who were treated with psoralen and ultraviolet $\mathrm{A},{ }^{11}$ whereas our results, from a substantially larger cohort, suggest that ciclosporin does not increase overall or cancer mortality risk, consistent with the previous report regarding mortality. ${ }^{2}$ The literature has been mixed regarding whether systemic corticosteroids increase the risk of mortality. ${ }^{5-10}$ Our results, which showed increased mortality when patients with systemic inflammatory co-morbidities are included but no increased mortality risk when they are excluded, support the view that systemic corticosteroid treatment does not itself increase the risk of overall or cancer mortality, but rather that the systemic disease that serves as the indication for such treatment increases mortality in these patients. Likewise, dapsone therapy does not seem to increase overall or cancer mortality.

Our study of about 500 patients with ocular inflammation treated with alkylating agents showed no significant increase in overall mortality. However, cancer mortality results were less reassuring. Cancer mortality was not increased to a statistically significant degree in either the fully adjusted within cohort comparison or the comparison with the general population, but in each case risk ratios were high and the $95 \%$ confidence intervals only narrowly included 1.0. Thus, our observations could be consistent with a moderate increase in overall cancer mortality-as suggested by a minority of previous reports ${ }^{12} 13$-but cannot be taken as conclusive without expansion of the cohort. Potentially increased cancer mortality - which would probably be cancelled out by survival benefits when alkylating agents are used to treat life threatening autoimmune diseases such as Wegener's granulomatosisis a clinically important consideration regarding the use of alkylating agents in non-lethal diseases such as ocular inflammation. Cyclophosphamide results were similar to the overall alkylating agent results. Additional data-especially regarding chlorambucil, for which we had a limited number of observations - are needed to better evaluate the overall and cancer mortality risk when these agents are given for inflammatory diseases.

Our data about the recently introduced TNF inhibitors were limited regarding the number of patients (about 200) and the duration of follow-up (median 2. 8 years). However, the large adverse effects we observed add to concerns that use of these agents may substantially increase overall and cancer mortality. A previous meta-analysis of randomised trial data, which showed increased risk of cancer with TNF inhibitor therapy with a dose-response pattern, ${ }^{14}$ is consistent with our findings. However, our findings are inconsistent with those of four large observational studies of patients with rheumatoid arthritis over substantially longer periods $s^{315-17}$ and one study of patients with Crohn's disease ${ }^{18}$ each of which found no increased overall cancer risk, with risk ratios close to 1.0. It has previously been proposed that TNF inhibitors may accelerate the clinical course of pre-existing but subclinical cancers, but may not initiate cancer. ${ }^{14}$ If so, an initially increased risk of clinically manifest cancer should be diluted with a longer duration of follow-up, which would be consistent with the available publications and our results. Readers should recognise that our TNF inhibitor results are less robust than the results for the other agents. A large proportion of our patients treated with TNF inhibitors had associated systemic inflammatory diseases which may have increased their mortality risk. We had limited ability to conduct critical sensitivity analyses to verify our results, and the results are of marginal statistical significance in a study where multiple comparisons were performed. Therefore, we do not believe these results to be conclusive. However, the apparent strength of the 


\section{WHAT IS ALREADY KNOWN ON THIS TOPIC}

Few data are available about whether overall mortality or cancer mortality is raised after treatment with immunosuppressive agents for inflammatory diseases

Some findings suggest that overall cancer incidence may be increased with alkylating agents and with tumour necrosis factor (TNF) inhibitors, but most reports suggest that it is not substantially raised with the other agents

\section{WHAT THIS STUDY ADDS}

Use of antimetabolites, T cell inhibitors, dapsone, and systemic corticosteroids does not seem to increase overall or cancer related mortality.

Use of alkylating agents was not associated with increased overall mortality, but cancer related mortality was non-significantly increased.

Preliminary data suggest that overall and cancer mortality may be increased with TNF inhibitors; these findings should be confirmed in additional studies in view of methodological limitations of our study for this drug class.

observed adverse effects indicates a need for additional evaluation of the potential risks of these widely used agents, including further evaluation of the possibility that TNF inhibitors and alkylating agents in combination or in succession increase mortality risk, as has been suggested in a previous report. ${ }^{26}$

In summary, our results suggest that methotrexate, azathioprine, mycophenolate mofetil, ciclosporin, dapsone, and systemic corticosteroids given to patients with ocular inflammatory diseases do not increase the risk of overall or cancer mortality. Alkylating agents did not significantly increase the risk of overall mortality, but our results could be consistent with a small to moderate increase in cancer mortality risk with these agents. Our findings suggesting substantially increased overall and cancer mortality after TNF inhibitor therapy should not be taken as conclusive unless supported by more data covering a longer period of time.

Contributors: Conception and design (JHK, ED, JPD, CSF, SG, KJH, DAJ, ROK, GAL-C, TL, RBN, SSP, JTR, EBS, JET); data analysis and interpretation (JHK, AH, KJH, CWN); acquisition of the data (JHK, ED, SG, ROK, TL, SSP); drafting the manuscript (JHK); critical revision of the manuscript for important intellectual content (all authors); final approval of the article (all authors); statistical analysis (JHK, CWN, AH); obtaining funding (JHK); administrative, technical, or material support (all authors); supervision (JHK, CSF, DAJ, GAL-C, RBN, JTR, EBS, JET). As senior author of the manuscript and principal investigator of the study, JHK had full access to all the data in the study and takes responsibility for the integrity of the data and the accuracy of the data analysis. JHK is independent of any commercial funder.

Funding: This study was supported primarily by National Eye Institute Grant EY014943 (JHK). Additional support was provided by Research to Prevent Blindness and the Paul and Evanina Mackall Foundation. JHK is an RPB James S Adams Special Scholar Award recipient. DAI and JTR are Research to Prevent Blindness Senior Scientific Investigator Award recipients. JET is an RPB Harrington Special Scholar Award recipient. GAL C was previously supported by and RBN continues to be supported by intramural funds of the National Eye Institute. None of the sponsors had any role in the design and conduct of the report; collection, management, analysis, and interpretation of the data; or in the preparation, review, and approval of this manuscript.

Competing interests: JTR has received $\leq \$ 10000$ for consulting fees or paid advisory boards for Abbott Laboratories (ciclosporin, adalimumab) and Novartis (ciclosporin), and has a research grant from Abbott Laboratories; EBS has received a research grant from Abbott Laboratories; DAJ has received $\leq \$ 10000$ for consulting fees or paid advisory boards for Novartis. JTR and EBS have received a research grant from Centocor (infliximab); CSF reports having received fees of $\leq \$$
10000 for speaking at the invitation of Centocor. JTR reports equity ownership/stock options >\$10 000 in Amgen (etanercept). JHK, JPD, CSF, JTR, and EBS previously participated in a clinical trial of a competing product, fluocinolone acetonide implant, sponsored by Bausch \& Lomb; JHK, JPD, CSF, DAJ, RBN, and JET are participating in an NIH-sponsored study comparing this product with systemic therapy for uveitis, and GALC previously participated in this trial; Bausch \& Lomb is providing a limited amount of drug product in support of this study.

Ethics approval: Institutional review board approval was granted and maintained at all participating sites throughout the study.

1 Kempen JH, Gangaputra S, Daniel E, Levy-Clarke GA, Nussenblatt RB, Rosenbaum JT, et al. Long-term risk of malignancy among patients treated with immunosuppressive agents for ocular inflammation: a critical assessment of the evidence. Am J Ophthalmol 2008;146:802-12.

2 Van den Borne BE, Landewe RB, Houkes I, Schild F, van der Heyden PC, Hazes JM, et al. No increased risk of malignancies and mortality in cyclosporin A-treated patients with rheumatoid arthritis. Arthritis Rheum 1998;41:1930-7.

3 Jacobsson LT, Turesson C, Nilsson JA, Petersson IF, Lindqvist E, Saxne T, et al. Treatment with TNF blockers and mortality risk in patients with rheumatoid arthritis. Ann Rheum Dis 2007;66:670-5.

4 Carmona L, Descalzo MA, Perez-Pampin E, Ruiz-Montesinos D, Erra A, Cobo T, et al. All-cause and cause-specific mortality in rheumatoid arthritis are not greater than expected when treated with tumour necrosis factor antagonists. Ann Rheum Dis 2007;66:880-5.

5 Wolfe F, Mitchell DM, Sibley JT, Fries JF, Bloch DA, Williams CA, et al. The mortality of rheumatoid arthritis. Arthritis Rheum 1994;37:481-94.

6 Pincus T, Callahan LF, Vaughn WK. Questionnaire, walking time and button test measures of functional capacity as predictive markers fo mortality in rheumatoid arthritis. J Rheumatol 1987;14:240-51.

7 Leigh JP, Fries JF. Mortality predictors among 263 patients with rheumatoid arthritis. J Rheumatol 1991;18:1307-12.

8 Sihvonen S, Korpela M, Mustonen J, Huhtala H, Karstila K, Pasternack A. Mortality in patients with rheumatoid arthritis treated with low-dose oral glucocorticoids. A population-based cohort study. Rheumatol 2006;33:1740-6.

9 Mitchell DM, Spitz PW, Young DY, Bloch DA, McShane DJ, Fries JF. Survival, prognosis, and causes of death in rheumatoid arthritis. Arthritis Rheum 1986;29:706-14.

10 Allebeck P, Rodvall Y, Allander E. Mortality in rheumatoid arthritis, particularly as regards drug use. Scand J Rheumatol 1985;14:102-8.

11 Paul CF, Ho VC, McGeown C, Christophers E, Schmidtmann B, Guillaume JC, et al. Risk of malignancies in psoriasis patients treated with cyclosporine: a 5 y cohort study. J Invest Dermatol 2003;120:211-6.

12 Baker GL, Kahl LE, Zee BC, Stolzer BL, Agarwal AK, Medsger TA Jr. Malignancy following treatment of rheumatoid arthritis with cyclophosphamide. Long-term case-control follow-up study. Am J Med 1987;83:1-9.

13 Baltus JA, Boersma JW, Hartman AP, Vandenbroucke JP. The occurrence of malignancies in patients with rheumatoid arthritis treated with cyclophosphamide: a controlled retrospective followup. Ann Rheum Dis 1983;42:368-73.

14 Bongartz T, Sutton AJ, Sweeting MJ, Buchan I, Matteson EL, Montori V. Anti-TNF antibody therapy in rheumatoid arthritis and the risk of serious infections and malignancies: systematic review and meta-analysis of rare harmful effects in randomized controlled trials. IAMA 2006;295:2275-85.

15 Fleischmann R, Baumgartner SW, Weisman MH, Liu T, White B, Peloso P. Long term safety of etanercept in elderly subjects with rheumatic diseases. Ann Rheum Dis 2006;65:379-84.

16 Setoguchi S, Solomon DH, Weinblatt ME, Katz JN, Avorn J, Glynn RJ, et al. Tumor necrosis factor alpha antagonist use and cancer in patients with rheumatoid arthritis. Arthritis Rheum 2006; 54:2757-64

17 Wolfe F, Michaud K. Biologic treatment of rheumatoid arthritis and the risk of malignancy: analyses from a large US observational study. Arthritis Rheum 2007:56:2886-95.

18 Biancone L, Orlando A, Kohn A, Colombo E, Sostegni R, Angelucci E, et al. Infliximab and newly diagnosed neoplasia in Crohn's disease: multicentre matched pair study. Gut 2006;55:228-33.

19 Kempen JH, Daniel E, Gangaputra S, Dreger K, Jabs DA, Kacmaz RO, et al. Methods for identifying long-term adverse effects of treatment in patients with eye diseases: the Systemic Immunosuppressive Therapy for Eye Diseases (SITE) cohort study. Ophthalmic Epidemiol 2008; 15:47-55.

20 Cowper DC, Kubal JD, Maynard C, Hynes DM. A primer and comparative review of major US mortality databases. Ann Epidemiol 2002;12:462-8 
21 Williams BC, Demitrack LB, Fries BE. The accuracy of the national death index when personal identifiers other than social security number are used. Am J Public Health 1992;82:1145-7.

22 Davis KB, Fisher L, Gillespie MJ, Pettinger M. A test of the national death index using the Coronary Artery Surgery Study (CASS). Contro Clin Trials 1985;6:179-91.

23 Sathiakumar N, Delzell E, Abdalla O. Using the national death index to obtain underlying cause of death codes. J Occup Environ Med 1998;40:808-13.
24 Cox DR, Oakes D. Analysis of survival data. London: Chapman and Hall, 1984.

25 Charlson ME, Pompei P, Ales KL, MacKenzie CR. A new method of classifying prognostic comorbidity in longitudinal studies: development and validation. J Chronic Dis 1987;40:373-83.

26 Stone JH, Holbrook JT, Marriott MA, Tibbs AK, Sejismundo LP, Min YI, et al. Solid malignancies among patients in the Wegener's

Granulomatosis Etanercept Trial. Arthritis Rheum 2006;54:1608-18.

Accepted: 13 March 2009 